\section{Case Reports in Neurology}

Case Rep Neurol 2017;9:267-271

\title{
MRI Signal Abnormalities of the Inferior Olivary Nuclei in Spinocerebellar Ataxia Type 2
}

\author{
Fumihito Yoshii, ${ }^{a, b}$ Hitoshi Tomiyasu ${ }^{c}$ Ryo Watanabe ${ }^{d}$ Masafuchi Ryo $^{b}$ \\ a Department of Neurology, Saiseikai Shonan Hiratsuka Hospital, Hiratsuka, Japan; \\ ${ }^{b}$ Department of Neurology, Tokai University Oiso Hospital, Oiso, Japan; \\ ${ }^{c}$ Hajyodo Tomiyasu Clinic, Kariya, Japan; ${ }^{d}$ Watanabe Clinic, Yokohama, Japan
}

\section{Keywords}

Spinocerebellar ataxia type $2 \cdot$ Magnetic resonance imaging $\cdot$ Inferior olivary nuclei $\cdot$ Hypertrophy

\begin{abstract}
Spinocerebellar ataxia type 2 (SCA2) is an autosomal dominant spinocerebellar degeneration, associated with extended repeats of the trinucleotide CAG in the ATXN2 gene on the long arm of chromosome 12. Magnetic resonance imaging (MRI) of SCA2 showed significant atrophies of the brainstem, middle cerebellar peduncles, and cerebellum. We report two genetically proven SCA2 patients who showed hypertrophy of the inferior olivary nuclei on proton density- and T2-weighted MRI. This pattern has never been reported in patients with SCA1, SCA3, or SCA6, and may make it possible to differentiate SCA2 from other hereditary spinocerebellar ataxias.

(C) 2017 The Author(s) Published by S. Karger AG, Basel
\end{abstract}

\section{Introduction}

Spinocerebellar ataxia type 2 (SCA2) is an autosomal dominant spinocerebellar degeneration characterized by progressive ataxia, slow saccadic eye movement, speech and swallowing difficulties, rigidity, bradykinesia, somatosensory deficits, and late intellectual deterioration. Sign and symptoms of disorder typically begin in mid-adulthood, but can appear anytime from childhood to late adulthood. Onset before the age of 20 years is correlated 


\section{Case Reports in Neurology}

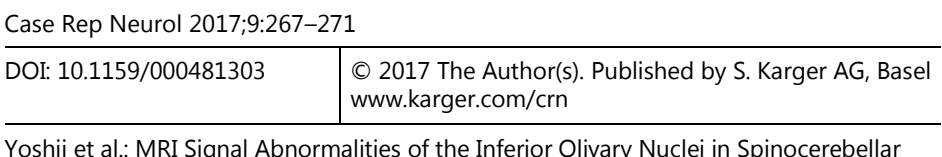
Ataxia Type 2

with a more rapid and severe course. In 1996, Sanpei et al. [1] and other groups [2, 3] demonstrated that in patients with SCA2, the ATXN2 gene on the long arm of chromosome 12 contained 35-59 repeats of the trinucleotide CAG, encoding ataxin-2 with an elongated polyglutamine tract, whereas only 15-24 repeats were present in healthy individuals. Thus, a clinical diagnosis of SCA2 can be made based upon detection of an increased number of CAG repeats in the ATXN2 gene. The extended ataxin-2 protein was reported to bind to the intracellular calcium release channel InsP(3)R1, altering neuronal excitability [4].

Previous magnetic resonance imaging (MRI) studies of SCA2 have disclosed significant atrophies of the pons, mesencephalon, middle cerebellar peduncles, and cerebellum $[5,6]$, which are consistent with typical changes of severe olivopontocerebellar atrophy [5]. However, these morphological changes often overlap with those in other autosomal dominant spinocerebellar ataxias, such as SCA1, SCA3, or SCA6. Here we report two patients with SCA2 who showed signal abnormalities of the inferior olivary nuclei on proton density (PD)- and T2-weighted MRI. This pattern has never been reported in other hereditary spinocerebellar ataxias, and may, we think, be relatively specific for SCA2.

\section{Case Presentation}

Patient 1 is a 37 -year-old man, who noticed difficulty in walking when he was 22 years old. His neurological condition gradually worsened and he showed irritability, slow mental response, and reduced physical performance at age 27 . He started to use a wheelchair at age 30 . When he was admitted to our hospital at age 37, he showed marked dysarthria, truncal and limb ataxia, slow saccadic eye movement, hyporeflexia, and distal dominant sensory disturbance. Palatal myoclonus was not observed during his course. Total intellectual quotient (IQ) score in the Wechsler Adult Intelligence Scale-Revised (WAIS-R) was 39. Similar ataxic symptoms were also observed in his grandfather and father. He was diagnosed as SCA2 with expanded CAG repeats; 22/43. Brain MRI was performed using a 1.5-T superconducting system (Magnetom, Siemens). T1-weighted images revealed marked atrophy in the brainstem, middle cerebellar peduncles or cerebellum. PD- and T2-weighted images showed hyperintense signals in the basal portion of the pons (transverse pontine fibers) and in the bilateral inferior olivary nuclei (Fig. 1a). These changes were more clearly seen in the PDweighted images. No ischemic lesions were found in the brainstem or cerebellum.

Patient 2 is a 20-year-old woman, who first noticed unsteadiness of gait at age 13 . She developed emotional instability and slow physical performance at age 17 . When she was admitted to our hospital at age 20 , she had explosive dysarthria, poor limb coordination, slow saccadic eye movements, hyporeflexia, and ataxia with a wide-based gait. Palatal myoclonus did not appear during her course. WAIS-R score (total IQ) was 40. Similar symptoms were observed in 2 other members of her family, over three generations. DNA analysis disclosed expanded CAG repeats $(22 / 51)$ in the region encoding SCA2. T1-weighted MRI revealed marked atrophy in the brainstem, middle cerebellar peduncles, or cerebellum, and T2-weighted images showed high signal intensities in the basal portion of the pons. Bilateral inferior olivary nuclei were enlarged and appeared as high signal intensities on PD-weighted images (Fig. 1b). There were no ischemic lesions in the brainstem or cerebellum. 


\section{Case Reports in Neurology}

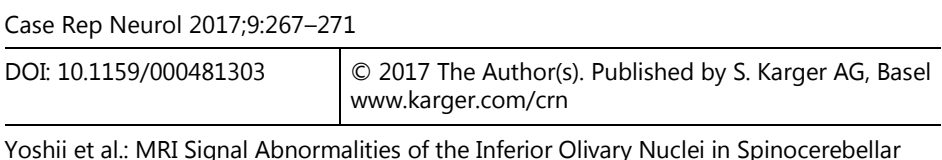

Ataxia Type 2

\section{Discussion}

Our two patients with SCA2, definitively diagnosed by DNA analysis, showed signal abnormalities of the bilateral inferior olivary nuclei on MRI. Neuropathologic examination of SCA2 patients consistently shows neuronal loss and gliosis in the pontine nucleus, cerebellar hemisphere, and vermis, as well as diffuse disappearance of myelinated fibers in the pontocerebellar and olivocerebellar pathways [5]. The inferior olivary nuclei exhibit severe neuronal cell loss and gliosis [7, 8]. Thus, abnormal signal intensities of the inferior olivary nuclei on MRI might simply reflect these pathological changes, and usually present clearly as increased signal intensities on T2-weighted images. However, in our patients, the signal abnormalities were more prominent on PD-weighted images, which depend primarily on the density of protons in the imaging volume, suggesting that the pathologic changes in the inferior olive were different from those in typical gliosis. Furthermore, the olivary nuclei seemed to be enlarged. So, we cannot completely exclude the possibility that the MRI findings might reflect hypertrophy or pseudohypertrophy (vacuolar degeneration of the enlarged neurons, hypertrophy of astrocytes, and fibrillary gliosis) of the inferior olive, which has been frequently reported in ischemic, hemorrhagic, and inflammatory lesions, or less frequently, traumatic lesions involving the Guillain-Mollaret triangle.

Similar olivary changes on MRI were sometimes observed in sporadic cases of olivopontocerebellar atrophy [9] or progressive supranuclear palsy (PSP) [10]. In PSP, inferior olivary hypertrophy is generally uncommon [11], but atrophy and fibrillary gliosis of the tegmentum of the pons, including that of the bilateral central tegmental tracts, are observed, particularly in patients with hypertrophic neurons in the olive [12]. A chronologic and morphometric study of the olivary nucleus has revealed that olivary hypertrophy or pseudohypertrophy after interruption of the central tegmental tract often appears prior to olivary atrophy [13], and an MRI-based volumetric study has disclosed that the brainstem volume was significantly reduced in SCA2 compared with SCA1, SCA3 [14], or SCA6 [15]. Therefore, we speculate that severe brainstem atrophy with secondary degeneration of the central tegmental tract, as reported in patients with PSP [10], may contribute to the development of the hypertrophy and signal abnormality in the inferior olive of some SCA2 patients.

\section{Conclusion}

Further studies are necessary to elucidate the appearance of the inferior olivary nuclei of SCA2 patients on MRI, especially in relation to age of onset, severity or duration of the disease, and other clinical features such as palatal myoclonus. However, our observations suggest that careful attention to the MR findings of the inferior olivary nuclei may allow differentiation of SCA2 from other hereditary spinocerebellar ataxias.

\section{Acknowledgements}

The authors thank Mrs. Isa Yoshinari for her technical assistance in manuscript preparation. 
Yoshii et al.: MRI Signal Abnormalities of the Inferior Olivary Nuclei in Spinocerebellar Ataxia Type 2

\section{Statement of Ethics}

The authors have no ethical conflicts to disclose.

\section{Disclosure Statement}

The authors declare that there is no conflict of interests regarding the publication of this article.

\section{References}

-1 Sanpei K, Takano H, Igarashi S, Sato T, Oyake M, Sasaki H, Wakisaka A, Tashiro K, Ishida Y, Ikeuchi T, Koide R, Saito M, Sato A, Tanaka T, Hanyu S, Takiyama Y, Nishizawa M, Shimizu N, Nomura Y, Segawa M, Iwabuchi K, Eguchi I, Tanaka H, Takahashi H, Tsuji S: Identification of the spinocerebellar ataxia type 2 gene using a direct identification of repeat expansion and cloning technique, DIRECT. Nat Genet 1993;5:277-284.

-2 Pulst SM, Nechiporuk A, Nechiporuk T, Gispert S, Chen XN, Lopes-Cendes I, Pearlman S, Starkman S, Orozco-Diaz G, Lunkes A, DeJong P, Rouleau GA, Auburger G, Korenberg JR, Figueroa C, Sahba S: Moderate expansion of a normally biallelic trinucleotide repeat in spinocerebellar ataxia type 2 . Nat Genet 1996;14:269-276.

-3 Imbert G, Saudou F, Yvert G, Devys D, Trottier Y, Garnier JM, Weber C, Mandel JL, Cancel G, Abbas N, Dürr A, Didierjean O, Stevanin G, Agid Y, Brice A: Cloning of the gene for spinocerebellar ataxia 2 reveals a locus with high sensitivity to expanded CAG/glutamine repeats. Nat Genet 1996;14:285-291.

-4 Liu J, Tang TS, Tu H, Nelson O, Herndon E, Huynh DP, Pulst SM, Bezprozvanny I: Deranged calcium signaling and neurodegeneration in spinocerebellar ataxia type 2. J Neurosci 2009;29:9148-9162.

5 Auberger GW: Spinocerebellar ataxia type 2. Handb Clin Neurol 2012;103:423-436.

-6 Klaes A, Reckziegel E, Franca MC Jr, Rezende TJ, Vedolin LM, Jardim LB, Saute JA: MR imaging in spinocerebellar ataxias: a systematic review. AJNR Am J Neuroradiol 2016;37:1405-1412.

-7 Dürr A, Smadja D, Cancel G, Lezin A, Stevanin G, Mikol J, Bellance R, Buisson GG, Chneiweiss H, Dellanave J, et al: Autosomal dominant cerebellar ataxia type I in Martinique (French West Indies). Clinical and neuropathological analysis of 53 patients from three unrelated SCA2 families. Brain 1995;118:1573-1581.

8 Seidel K, Siswanto S, Brunt ER, den Dunnen W, Korf HW, Rüb U: Brain pathology of spinocerebellar ataxias. Acta Neuropathol 2012;124:1-21.

-9 Savoiardo M, Grisoli M, Girotti F, Testa D, Caraceni T: MRI in sporadic olivopontocerebellar atrophy and striatonigral degeneration. Neurology 1997;48:790-792.

10 Yagishita A, Oda M: Progressive supranuclear palsy: MRI and pathological findings. Neuroradiology 1996;38:S60-S66.

-11 Katsuse 0, Dickson DW: Inferior olivary hypertrophy is uncommon in progressive supranuclear palsy. Acta Neuropathol 2004;108:143-146.

12 Hanihara T, Amano N, Takahashi T, Itoh Y, Yagishita S: Hypertrophy of the inferior olive in patients with progressive supranuclear palsy. Eur Neurol 1998;39:97-102.

13 Goto N, Kaneko M: Olivary enlargement. Chronological and morphometric analyses. Acta Neuropathol (Berg) 1981;54:275-282.

14 Klockgether T, Skalej M, Wedekind D, Luft AR, Welte D, Schulz JB, Abele M, Bürk K, Laccone F, Brice A, Dichgans J: Autosomal dominant cerebellar ataxia type 1. MRI-based volumetry of posterior fossa structures and basal ganglia in spinocerebellar ataxia types 1, 2 and 3. Brain 1998;121:1687-1693.

-15 Eichler L, Bellenberg B, Hahn HK, Köster O, Schöls L, Lukas C: Quantitative assessment of brain stem and cerebellar atrophy in spinocerebellar ataxia types 3 and 6: impact on clinical status. AJNR Am J Neuroradiol 2011;32:890-897. 


\section{Case Reports in Neurology}

Yoshii et al.: MRI Signal Abnormalities of the Inferior Olivary Nuclei in Spinocerebellar Ataxia Type 2

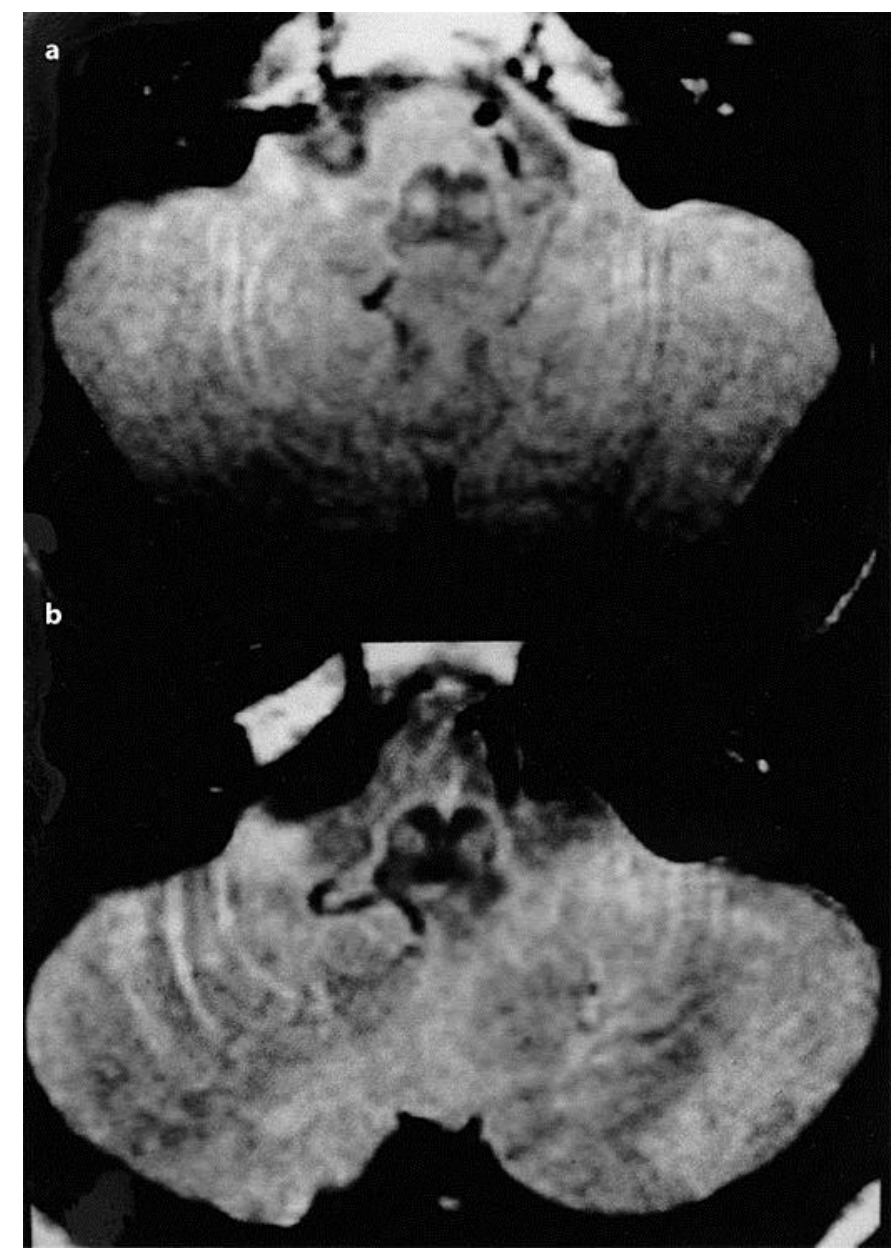

Fig. 1. Proton density-weighted MRI (TR 3,500 ms, TE $19 \mathrm{~ms}$ ) shows high signal intensity in the inferior olivary nuclei of patient 1 (a) and patient 2 (b). 